\title{
对苯二甲酰氯桥联 $\beta$-环糊精的合成及其对染料分子的协同键合
}

\author{
彭龙刘慧君* 胡趁孙允凯龙 威 \\ (南华大学化学化工学院 衡阳 421001)
}

\begin{abstract}
摘要 实验以 $\beta$-环糊精和对苯二甲酰氯为原料, 合成了一种新型刚性结构的桥联环糊精一一 $1,4-$ 二甲酰胺基苯桥联 $\beta$ 环糊精, 并通过 ${ }^{1} \mathrm{H} N \mathrm{NR}$ 、红外和紫外以及元素分析等对其结构进行了表征. 采用荧光光谱滴定法分别测定了该桥联环 糊精与几种具有不同形状和电子密度的染料分子在 $25{ }^{\circ} \mathrm{C}$ 磷酸缓冲溶液 $(\mathrm{pH}=7.20)$ 中的包结配位稳定常数 $K_{\mathrm{S}}$. 结果表 明, 桥联环糊精的键合能力远远增强, 桥联环糊精与天然 $\beta$-环糊精相比, 对 TNS, ANS, AR, NR 和 RhB 的包结稳定常数 分别提高了 3.3, 15.3, 2.2, 12.5 和 5.8 倍. 从主-客体间的尺寸匹配关系和多重识别机理等方面探讨了桥联环糊精对客体 分子的协同键合作用.
\end{abstract}

关键词 桥联 $\beta$-环糊精; 协同键合; 染料; 分子识别; 超分子化学

\section{Terephthaloyl Chloride Bridged Bis( $\beta$-cyclodextrin) and Their Synergetic Bonding Behaviors with Dyes}

\author{
Peng, Long Liu, Huijun* Hu, Cheng Sun, Yunkai Long, Wei \\ (College of Chemical Engineering, University of South China, Hengyang 421001)
}

\begin{abstract}
A novel bridged bis( $\beta$-cyclodextrin) with rigid aromatic diamino tethers were synthesized by the reaction of terephthaloyl chloride with $\beta$-cyclodextrin. The novel bridged bis( $\beta$-cyclodextrin) was analyzed by ${ }^{1} \mathrm{H}$ NMR, IR, UV and elemental analysis. The complex stability constants $\left(K_{\mathrm{S}}\right)$ for the inclusion complexation of these bis $(\beta$-cyclodextrin) with several dyes possessing different shapes and electron intensities were determined by means of fluorescence and UV-Vis titrations at $25{ }^{\circ} \mathrm{C}$ in phosphate buffer solution $(\mathrm{pH}=7.2)$. The results show that the bridged bis $(\beta$-cyclodextrin) showed much higher binding ability. Compared with native $\beta$-cyclodextrin, the binding abilities of the bridged bis( $\beta$-cyclodextrin) upon inclusion complexation to TNS, ANS, AR, NR and RhB were increased 3.3, 15.3, 2.2, 12.5, and 5.8 times, respectively. The cooperative binding ability of the bis( $\beta$-cyclodextrin) was discussed from the viewpoints of size/shape-fit interaction and multiple recognition mechanism.
\end{abstract}

Keywords bridged bis( $\beta$-cyclodextrin); synergetic bonding; dyes; molecular recognition; supramolecular chemistry

环糊精 $($ Cyclodextrins, 通常简称为 $\mathrm{CD}$ ) 是一类由 $D$ 吡喃葡萄糖单元首尾连成环的大环化合物, 是继冠醚以 后超分子化学研究的第二代主体化合物. 其环内疏水空 腔可以在溶液和固相中包结多种无机、有机和生物分子 形成主一客体包合物或超分子配合物，因此被广泛应用 于分子识别、分子组装、药物载体和模拟酶等诸多领域 [1 4]. 然而, 天然环糊精与客体分子的结合作用一般较 弱 ${ }^{[5,6]}$, 即使在天然环糊精边臂上引入某种功能基的简 单修饰也难在分子键合能力上达到显著的提高 ${ }^{[7]}$, 进而 使其在类酶研究方面受到一定限制, 而将环糊精单元通 过各种各样功能基或特定桥链基团连接之后, 两个相邻
的环糊精空腔有可能协同参与对形状和尺寸适合的客 体分子的包结配位作用，从而显著提高母体环糊精对客 体分子的键合能力和选择性. 尽管早在 20 世纪 70 年代 就合成了桥联环糊精, 而对这一类化合物的分子识别和 模拟酶研究则在 20 世纪 50 年代以后, 特别是最近 10 来年才得到深入研究, 美国的 Breslow 教授、荷兰的 Nolte 教授和日本的 Fujita 教授对这方面的研究作出了 重要贡献 ${ }^{[8,9]}$. 另外, 当桥联环糊精的桥链上有合适的取 代基时，可以实现对客体分子的三重识别或多重识别， 即在桥联环糊精的两个环糊精空腔协同包结底物的同 时, 桥链上的某些基团对客体分子也产生识别作

*E-mail: liuhuijun@usc.edu.cn

Received November 7, 2014; revised January 25, 2015; published online February 28, 2015.

Project supported by the National Natural Science Foundation of China (Nos. 11375084, 11305086).

国家自然科学基金(Nos. 11375084, 11305086 )资助项目. 
用 ${ }^{[10,11]}$. 因此拥有各种不同功能基的桥联环糊精的合成 和分子识别研究也变得越来越引人注目 ${ }^{[12 \sim 14]}$.

芳香二胺桥联环糊精由于在桥链上引入了刚性较 强的芳香基团, 不仅增强了微环境的疏水性, 而且扩展 了环糊精疏水的空腔, 因此更利于环糊精对疏水性客体 分子的包结 ${ }^{[15]}$. 本工作合成了一种新型的芳香桥联环 糊精——1,4-二甲酰胺基苯桥联 $\beta$-环糊精(Scheme 1), 通过 ${ }^{1} \mathrm{H}$ NMR、红外、紫外以及元素分析等表征手段, 并 选用几种符合桥联环糊精配位研究要求的典型染料作 为客体, 既有线型分子 $\mathrm{AR}, \mathrm{NR}, \mathrm{TNS}$ 和 $\mathrm{ANS}$, 又有 $\mathrm{T}$ 型 结构的 $\mathrm{RhB}$ 分子, 用苂光光谱滴定法测定了天然环糊 精和新型的桥联环糊精 $\mathbf{1}$ 与这些客体分子在磷酸缓冲溶 液 $(\mathrm{pH}=7.20)$ 中 $25{ }^{\circ} \mathrm{C}$ 时的包结稳定常数. 实验结果表 明, 桥联 $\beta$-环糊精 1 不仅大大扩展了母体环糊精原有的 分子键合能力, 而且能够识别出客体分子尺寸与结构上 的差异. 本文从主-客体间的尺寸匹配关系和多点识别 等方面探讨了桥联环糊精的协同识别作用机理. 主一客 体化合物的结构如图 1 所示.

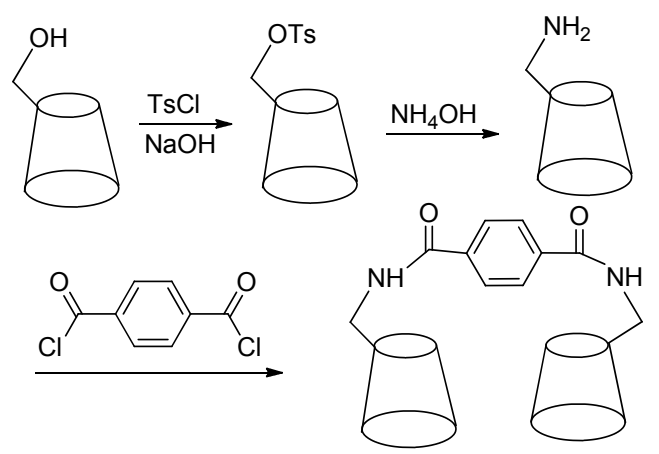

图式 1 主体 1 的合成路线

Scheme 1 The synthetic route of host 1<smiles>O=C(NCC1C2CC3CC(C2)C1C3)c1ccc(C(=O)NCC23CC4CC(C2)C(C4)C3)cc1</smiles>

ANS

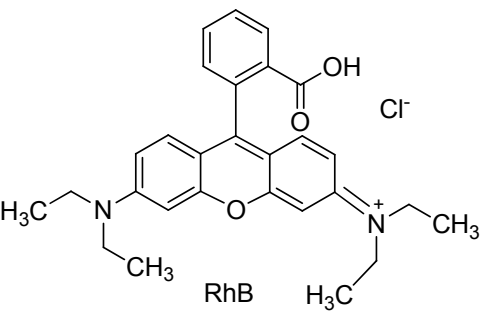<smiles>Cc1ccc(Nc2ccc3cc(S(=O)(=O)O[Na])ccc3c2)cc1</smiles><smiles></smiles>

图 1 主-客体分子的结构

Figure 1 Molecular structures of hosts and guests

\section{1 结果与讨论}

\section{1 荧光光谱滴定}

为定量研究主一客体间的键合行为, 采用苂光光谱 测定客体 AR, NR, RhB, ANS 和 TNS 与主体环糊精的包 结配位稳定常数 $\left(K_{\mathrm{S}}\right)$ 和 Gibbs 自由能变化值 $\left(\Delta G^{0}\right)$. 从图 2 中可以看到, 加入主体桥联环糊精 1 后, NR 的相对苂 光强度随主体化合物 $\mathbf{1}$ 的加入逐渐增加, 此现象与所研 究的 ANS, TNS 和 AR 表现出来的苂光行为一致, 表明 客体染料分子由极性环境进入到环糊精疏水空腔的非 极性环境中，形成了超分子配合物.

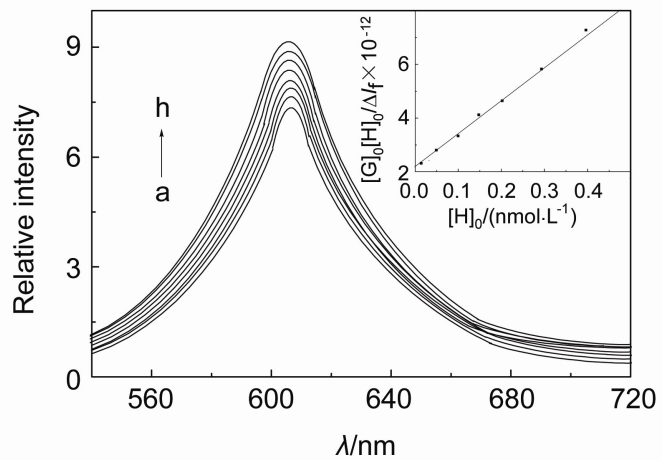

图 2 在 $\mathrm{pH}=7.20$ 的缓冲溶液中 $\mathrm{NR}$ 随主体 $\mathbf{1}$ 加入后的荧光 光谱变化图

Figure 2 Fluorescence spectral changes of NR upon addition of host $\mathbf{1}$ in aqueous buffer solution at $\mathrm{pH}=7.20$

Inset is the liner correlation for complexation of NR with host $\mathbf{1}$. The concentration of NR is $4.0 \mu \mathrm{mol} / \mathrm{L}$. The concentration of host $\mathbf{1}$ is 0,20 , $50,100,150,200,300,400 \mu \mathrm{mol} / \mathrm{L}$ from a to h, respectively. Excitation wavelength is $520 \mathrm{~nm}$

与此相反, 如图 3 所示. 主体化合物 1 的加入却使 $\mathrm{RhB}$ 荧光猝灭, 这与天然 $\beta$-环糊精和单修饰 $\beta$-环糊精能 使 RhB 苂光猝灭的现象相似，而同为桥联环糊精，联吡 啶桥联 $\beta$-环糊精对 $\mathrm{RhB}$ 客体分子的荧光有增感作用 ${ }^{[16]}$. 这可能是由于联吡啶桥联环糊精中的桥链可以使两个 环糊精空腔对 RhB 的两性离子构型产生最为有效的键 合, 固定客体的构型, 从而提高客体分子的苂光量子产 率. 而对于主体化合物 $\mathbf{1}, \mathrm{RhB}$ 可能是以无色内酯的形 式通过形成氢键进行包结，这种结构在可见光区没有荧 光，从而导致包结后其苂光猝灭.

桥联环糊精与客体分子包结作用的配位比通过等 摩尔连续变化法(Job's 方法)确定. 具体方法为固定主体 $\mathrm{H}$ 和客体 $\mathrm{G}$ 的总物质的量不变, 连续改变两种组分的比 例, 测量各组混合液的苂光发射(或紫外吸收)强度与相 同条件下客体自身苂光发射(或紫外吸收)强度的差值 $\Delta I_{\mathrm{f}}$ (或 $\left.\Delta A\right)$, 以 $\Delta I_{\mathrm{f}}$ (或 $\Delta A$ ) 对任一组分的摩尔分数作图, 可以得到主-客体包结配位的化学计量比. 图 4 显示 $1 / \mathrm{NR}$ 体系的 Job's 曲线, 该曲线在摩尔分数 0.5 处出现 


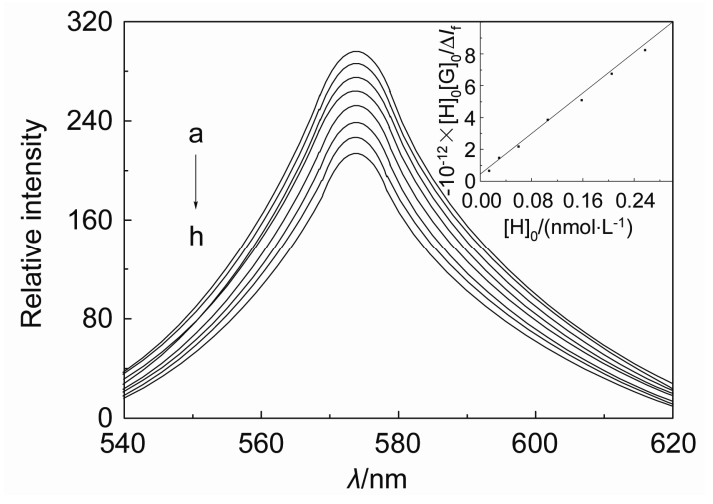

图 3 在 $\mathrm{pH}=7.20$ 的缓冲溶液中 $\mathrm{RhB}$ 随主体 $\mathbf{1}$ 加入后的荧光 光谱变化图

Figure 3 Fluorescence spectral changes of $\mathrm{RhB}$ upon addition of host $\mathbf{1}$ in aqueous buffer solution at $\mathrm{pH}=7.20$

Inset is the liner correlation for complexation of $\mathrm{RhB}$ with host $\mathbf{1}$. The concentration of $\mathrm{RhB}$ is $1.8 \mu \mathrm{mol} / \mathrm{L}$. The concentration of host 1 is 0,20 , $40,80,100,160,220,280 \mu \mathrm{mol} \cdot \mathrm{L}^{-1}$ from a to $\mathrm{h}$, respectively; Excitation wavelength is $525 \mathrm{~nm}$

最大值，说明桥联环糊精 1 与 NR 形成了计量比为 $1: 1$ 的包结配合物.

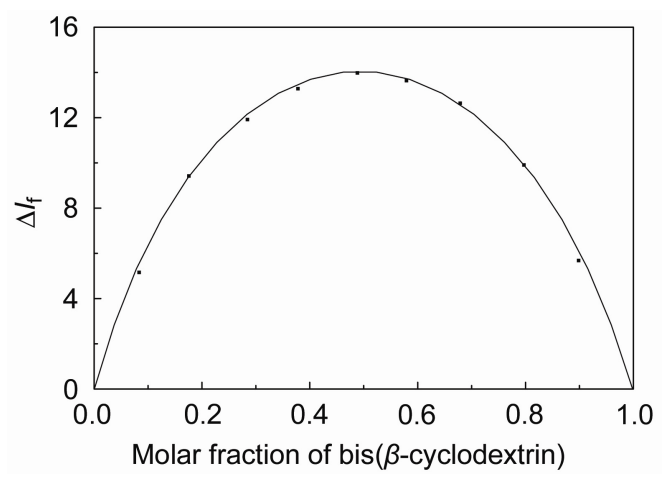

图 $41 / \mathrm{NR}$ 体系 Job 曲线

Figure 4 Job plots of $1 / \mathrm{NR}$ system

$[$ bis $(\beta$-cyclodextrin $)]+[\mathrm{NR}]=4.0 \times 10^{-5} \mathrm{~mol} \cdot \mathrm{L}^{-1}$

此外，其它的主一客体包结配位过程也都显示了 $1: 1$ 的化学计量比. 将主体 1 中的两个 $\beta$-环糊精空腔视 为一个主体单元，则主一客体间的包结配位作用过程可 表示为:

$$
\mathrm{H}+\mathrm{G} \stackrel{K_{\mathrm{s}}}{\rightleftharpoons} \mathrm{G} \cdot \mathrm{H}
$$

其中 $\mathrm{H}$ 表示主体, $\mathrm{G}$ 表示客体. 在光谱滴定实验中, 环 糊精及其衍生物的浓度远远大于客体的浓度. 即 $[\mathrm{H}]_{0} \gg$ $[\mathrm{G}]_{0}$. 因此, 超分子体系的稳定常数可以根据修饰的 Benesi-Hildebrand 方程进行计算 ${ }^{[15]}$ :

$$
[\mathrm{G}]_{0}[\mathrm{H}]_{0} / \Delta I_{\mathrm{f}}=\left(1 / K_{\mathrm{S}} \alpha\right)+[\mathrm{H}]_{0} / \alpha
$$

式中 $[\mathrm{H}]_{0},[\mathrm{G}]_{0}$ 分别为主体环糊精衍生物和客体染料分 子的初始浓度. $\Delta I_{\mathrm{f}}$ 为加入主体前后染料的光谱相对强度
变化, 即 $\Delta I_{\mathrm{f}}=I_{\mathrm{f}}$ (有主体) $-I_{\mathrm{f}}$ (无主体), $\alpha$ 为光谱的敏感因 子. 如图 2 插图和图 3 插图所示, 在所检验的客体分子 中, 以 $[\mathrm{G}]_{0}[\mathrm{H}]_{0} / \Delta I_{\mathrm{f}}$ 值对 $[\mathrm{H}]_{0}$ 作图，均给出了优良的线性 关系，进一步证实了假定的配合物 $1: 1$ 化学计量是正 确的. 从截距和斜率计算出的超分子体系稳定常数 $\left(\lg K_{\mathrm{S}}\right)$ 和自由能变化 $\left(\Delta G^{0}\right)$ 均列入了表 1 , 为了进行比较, 已报道的天然环糊精与染料客体分子包结配位的稳定 常数和自由能变化值也列入表 1 中.

表 $1 \beta$-环糊精和桥联 $\beta$-环糊精主体 1 与染料在 $25{ }^{\circ} \mathrm{C}$ 的缓冲 溶液 $(\mathrm{pH}=7.20)$ 中形成 $1: 1$ 超分子配合物的稳定常数 $\left(K_{\mathrm{S}}\right)$ 和 吉布斯自由能 $\left(-\Delta G^{0}\right)$ 值

Table 1 Complex stability constant $\left(K_{\mathrm{S}}\right)$ and Gibbs free energy change $\left(-\Delta G^{0}\right)$ for $1: 1$ inclusion complexation of dyes with $\beta$-cyclodextrin and bis $(\beta$-cyclodextrin) 1 in aqueous buffer solu-

\begin{tabular}{|c|c|c|c|c|c|}
\hline Host & Guest & $K_{\mathrm{S}}$ & $\lg K_{\mathrm{S}}$ & $-\Delta G^{0} /\left(\mathrm{kJ} \cdot \mathrm{mol}^{-1}\right)$ & Ref. \\
\hline \multirow{5}{*}{$\beta-\mathrm{CD}$} & TNS & 3670 & 3.56 & 20.35 & {$[18]$} \\
\hline & ANS & 103 & 2.01 & 11.49 & [18] \\
\hline & $\mathrm{AR}$ & 2630 & 3.42 & 19.50 & [19] \\
\hline & NR & 480 & 2.78 & 15.87 & {$[20]$} \\
\hline & $\mathrm{RhB}$ & 4240 & 3.63 & 20.70 & {$[21]$} \\
\hline \multirow{5}{*}{1} & TNS & 12100 & 4.08 & 23.12 & This work \\
\hline & ANS & 1570 & 3.19 & 17.63 & This work \\
\hline & AR & 5786 & 3.76 & 21.50 & This work \\
\hline & NR & 6000 & 3.78 & 21.26 & This work \\
\hline & $\mathrm{RhB}$ & 24590 & 4.39 & 24.77 & This work \\
\hline
\end{tabular}
tion $(\mathrm{pH}=7.20)$ at $25{ }^{\circ} \mathrm{C}$

\section{3 分子键合能力}

天然和简单修饰 $\beta$-环糊精与模型底物的包结配位 只能提供较低的键合常数. 但是, 从表 1 可以看出, 桥 联环糊精 1 与天然 $\beta$-环糊精相比, 对 TNS, ANS, AR, NR 和 $\mathrm{RhB}$ 的包结稳定常数分别提高了 $3.3,15.3,2.2,12.5$ 和 5.8 倍. 这主要应归于桥联环糊精 $\mathbf{1}$ 中两个疏水空腔 对客体染料分子的协同键合作用，从而提高了母体环糊 精原有的分子键合能力.

在所探究的客体分子中, 桥联双环糊精 1 对非线形 客体分子 $\mathrm{RhB}$ 给出了明显高于线形客体分子 $\mathrm{NR}$, TNS 等的键合能力. 桥联环糊精这种对客体分子形状的识别 能力主要应归因于桥链与客体分子之间的 $\pi-\pi$ 或氢键相 互作用. 分子力学研究已经证明, 桥联环糊精 1 在包结 线形客体分子时只有两个环糊精空腔参与了协同键合, 因此只给出了相对较低的键合常数. 而在键合非线形客 体分子 $\mathrm{RhB}$ 时，除两个环糊精空腔的协同键合外，桥链 基团也与客体分子的支链产生 $\pi-\pi$ 或氢键相互作用，从 而导致主-客体间强的键合能力.

从表 1 可以看出, 虽然 TNS 与 ANS 具有类似的结 构(二者都有菜环的结构), 但是不论是同天然环糊精还 是桥联环糊精进行反应，对这两种客体分子的键合能力 
却截然不同. 推测的可能原因是 TNS 的取代基在 2 位和 6 位, 而 ANS 的取代基在 1 位和 8 位, 在进入环糊精空 腔时, ANS 的取代基产生的位阻比 TNS 要大得多, 这样 在形成包结配合物的过程中, 具有构型优势的客体分子 TNS 都比 ANS 更容易被包结进入环糊精的疏水空腔形 成稳定的超分子配合物, 因而对 TNS 表现出相对较强 的键合能力, 天然环糊精对 TNS 的包结稳定常数是 ANS 的 36 倍, 证明了主客体的尺寸/形状匹配成为影响 其键合行为的主导因素. 然而, 由于桥连环糊精 1 可以 通过增加的一个空腔实现对客体的双重识别, 大大增强 了对 ANS 的键合能力, 给出了 15.7 倍于天然环糊精的 配位稳定常数. 而对于原本就相对稳定键合的 TNS, 这 种双重识别仅仅使其键合稳定常数增加了 3.3 倍而已.

另外，对于客体分子 AR 和 NR, 尽管其结构相似, 都具有类似葱的杂环且都是线形分子, 但天然环糊精对 它们的键合能力有较大的差异. 天然环糊精对 AR 的键 合比对 NR 强, 这可能是因为 AR 上的取代基相对较小, 从而能更容易地进入环糊精疏水空腔的缘故. 然而桥联 环糊精 1 却大大扩展了对 NR 的键合能力 (配合稳定常数 提高了 12.5 倍), 从而对两者的键合能力则给出了相反 的顺序 $\left(K_{\mathrm{NR}-1}>K_{\mathrm{AR}-1}\right)$. 这一结果表明, 在桥联环糊精 $\mathbf{1}$ 与这两种客体染料分子形成配合物时, 空间作用并不是 最主要的因素. 可能的原因是, AR 与 NR 带有的正电中 心与桥联环糊精 1 上的桥链负离子发生了静电相互作 用, 取代基的影响相对减弱, 因而使主体环糊精 1 对两 种客体分子 $\mathrm{AR}$ 和 $\mathrm{NR}$ 配位稳定常数较接近.

\section{2 结论}

桥联环糊精与尺寸适合的客体分子在水溶液中可 以包结配位, 使其能形成稳定的 $1: 1$ 超分子配合物, 这 种协同作用的结果使桥联环糊精对客体分子的包结能 力和分子选择性明显强于天然环糊精. 换言之, 就是两 个环糊精单元对同一客体的双重疏水相互作用. 这种特 性使其有可能在酶-底物和抗原-抗体强键合相互作用 模拟研究中得到重要应用. 另外, 如桥联环糊精 1 对 $\mathrm{RhB}$ 的键合能力和对 $\mathrm{RhB} / \mathrm{AR}$ 的分子选择性分别达到天 然环糊精的 5.8 倍和 4.2 倍. 主一客体间的尺寸/形状匹配 关系, 分子间氢键相互作用以及静电相互作用是决定桥 联环糊精 1 与客体染料分子包结配位稳定性的重要因 素. 这对于设计结构新颖、对客体分子具有高键合能力 和分子选择性的桥联环糊精主体具有实际的指导意义.

\section{3 实验部分}

\section{1 试剂}

$\beta$-环糊精(天津市大茂化学试剂厂, 生物试剂)用蒸
馏水重结晶两次, $60{ }^{\circ} \mathrm{C}$ 下真空干燥 $24 \mathrm{~h}$ 后使用; 对甲苯 磺酰氯(天津市大茂化学试剂厂, 分析纯); $N, N$-二甲基 甲酰胺(天津市大茂化学试剂厂,分析纯); 氢氧化钠(天 津市大茂化学试剂厂, 分析纯); 氨水(分析纯，长沙延 风化学试剂有限公司); 对苯二甲酰氯(上海金山亭新化 工厂, 分析纯); 2-对甲苯胺基-6-䒬磺酸钠(TNS)、听啶红 (AR)、1-苯胺基-8-菜磺酸铵(ANS)和亮绿(BG)均为日本 和光公司产品; 中性红(NR)天津市福晨化学试剂厂产 品. 将 $4.37 \mathrm{~g}$ 二水合磷酸二氢钠和 $25.79 \mathrm{~g}$ 十二水合磷酸 氢二钠溶于 $1000 \mathrm{~mL}$ 二次蒸馏水中配制成 $\mathrm{pH}=7.20$ 的 缓冲溶液, 作为光谱滴定的溶剂.

\section{2 仪器及方法}

721 型紫外可见分光光度计, 天津市普瑞斯仪器有 限公司; Shimadu IRPrestige-21 红外光谱仪, 日本岛津公 司; Bruker AV-III 300MHZ 型核磁共振波谱仪, 瑞士 Bruker BioSpin 公司; LS45 荧光分光光度计, 日本岛津 公司. 在实验中，固定客体的浓度在 $2.5 \times 10^{-6} \sim 1 \times$ $10^{-5} \mathrm{~mol} / \mathrm{L}$ 范围内, 改变主体化合物的浓度, 观测不同 浓度的主体存在下客体荧光光谱的变化. 苂光光谱在 LS45 荧光分光光度计上用普通样品池 $(10 \mathrm{~mm} \times 10$ $\mathrm{mm} \times 45 \mathrm{~mm}$ ) 测定, 光栅狭缝宽度为 $10 \mathrm{~nm}$, 测定温度 由循环冷凝水控制在 $(25.0 \pm 0.1){ }^{\circ} \mathrm{C}$.

\section{3 桥联环糊精的合成}

\subsection{1 磺酰化 $\beta$-环糊精(6-OTs- $\beta$-CD) 的制备}

按文献[18]制备, 称取 $\beta$-环糊精 $5 \mathrm{~g}$ 加入到 $250 \mathrm{~mL}$ 圆底烧瓶中，同时加入 $1.8 \mathrm{~g} \mathrm{NaOH}$ 和 $100 \mathrm{~mL}$ 水，搅拌 至完全溶解, 加入冰块调节温度到 $0 \sim 5{ }^{\circ} \mathrm{C}$, 向其中加 入 $1.26 \mathrm{~g}$ 对甲苯磺酰氯( $\mathrm{TsCl})$, 搅拌反应 $5 \mathrm{~h}$, 过滤掉未 反应的 $\mathrm{TsCl}$, 用浓度为 $1 \mathrm{~mol} / \mathrm{L}$ 的 $\mathrm{HCl}$ 将滤液 $\mathrm{pH}$ 调为 $6 \sim 7$ 后放入冰箱 $4{ }^{\circ} \mathrm{C}$ 下冷藏 $24 \mathrm{~h}$, 会有白色固体析出, 过滤出固体并用蒸馏水重结晶两次, 再用乙腈一水混合 溶剂(体积比为 $1: 1$ )重结晶一次, 烘干后得纯净的白色 固体磺酰化 $\beta$-环糊精 (6-OTs- $\beta$-CD), 产率约为 $27 \%$. $R_{\mathrm{f}}=0.426$; FT-IR (KBr) $v: 3346,1647,1364,1159,1080$, $1028,837,815,580 \mathrm{~cm}^{-1}$, 与文献结果相符.

3.3.2 6-氨基 6-脱氧- $\beta$-环糊精(氨基环糊精)的合成制 备

按文献[22]制备, 取 $1 \mathrm{~g}$ 磺酰化 $\beta$-环糊精加入到 250 $\mathrm{mL}$ 圆底烧瓶中，同时量取 $25 \mathrm{~mL} 25 \%$ 的氨水加入烧瓶 中, 控制反应温度在 $50{ }^{\circ} \mathrm{C}$, 搅拌反应 $12 \mathrm{~h}$, 然后减压蒸 馏, 得白色固体. 固体干燥后溶于少量的水中, 经 Sephedex G-25 柱分离, 产率约为 31\%. 产品为白色粉 末. $R_{\mathrm{f}}=0.2$; FT-IR (KBr) $v: 3439,1641,1400,1193,1124$, $1034,601 \mathrm{~cm}^{-1}$, 与文献结果相符. 


\subsubsection{1,4-二甲酰胺基苯桥联 $\beta$-环糊精(1)的合成}

将 $0.5 \mathrm{~g}$ 氨基环糊精溶解在 $60 \mathrm{~mL} \mathrm{DMF}$ 中, 加入 $0.0447 \mathrm{~g}$ 对苯二甲酰氯, 控制温度在 $70{ }^{\circ} \mathrm{C}$, 反应 $24 \mathrm{~h}$, 然后加入 $100 \mathrm{~mL}$ 丙酮, 有白色沉淀析出, 过滤出沉淀, 再用丙酩冲洗去除 DMF. 经 Sephedex G-25 柱分离, 产 率 $15 \%$, 产品为白色晶体. UV-Vis $\left(\mathrm{H}_{2} \mathrm{O}\right) \lambda_{\max }[\varepsilon /(\mathrm{L} \cdot$ $\left.\mathrm{mol}^{-1} \cdot \mathrm{cm}^{-1}\right)$ ]: 204 (20251), 256 (13782) nm; ${ }^{1} \mathrm{H}$ NMR $\left(\mathrm{D}_{2} \mathrm{O}, 300 \mathrm{MHz}\right) \delta: 3.3 \sim 4.1(\mathrm{~m}, 63 \mathrm{H}), 4.9 \sim 5.1(\mathrm{~m}, 10 \mathrm{H})$, 7.9 (s, ArH, 4H); FT-IR (KBr) v: 1641.4, 1564.3, 1371.4, 1155.4, 1080.1, 1030.0, 947.1, 754.2, 706.0, $580.6 \mathrm{~cm}^{-1}$. Anal. calcd for $\mathrm{C}_{92} \mathrm{H}_{144} \mathrm{O}_{70} \mathrm{~N}_{2} \bullet 8 \mathrm{H}_{2} \mathrm{O}$ : C 43.46, H 5.91, N 1.10; found $\mathrm{C} 43.08, \mathrm{H} 6.15, \mathrm{~N} 1.14$.

辅助材料(Supporting Information) 合成主体化合物 的红外光谱图、天然 $\beta$-环糊精的红外光谱图和环糊精与 对苯二甲酰氯的机械混合物红外光谱图. 这些材料可以 免费从本刊网站(http://sioc-journal.cn/)上下载.

\section{References}

[1] Zhou, D.-X.; Sun, T.; Deng, W. Chin. J. Org. Chem. 2012, 32, 239 (in Chinese). (周冬香, 孙涛, 邓维, 有机化学, 2012, 32, 239.)

[2] Wan, J.-M.; Hu, Z.-W.; Chen, W.-X.; Zheng, S.-R.; Lü, S.-S.; Zhang, L. Acta Phys.-Chim. Sin. 2006, 22, 244 (in Chinese). (万军民, 胡智文, 陈文兴, 郑世战, 吕慎水, 张利, 物理化学学 报, 2006, 22, 244.)

[3] Abdel-Naby, M. A.; El-Refai, H. A.; Abdel-Fattah, A. F. J. Appl. Microbiol. 2011, 111, 1129.

[4] Thombre, R. S.; Kanekar, P. P. J. Microbiol. Biotechnol. Res. 2013, $3,57$.

[5] Chen, Y.; Han, N.; Yang, H.; Liu, Y. Acta Chim. Sinica 2007, 65, 1076 (in Chinese). (陈涌, 韩宁, 杨华, 刘育, 化学学报, 2007, 65, 1076.)

[6] Shen, H.-M.; Ji, H.-B.; Wu, H.-K.; Shi, H.-X. Chin. J. Org. Chem. 2014, 34, 1549 (in Chinese).
(沈海民, 纪红兵, 武宏科, 史鸿釒金, 有机化学, 2014, 34, 1549.)

[7] Ueno, A.; Ikeda, A.; Ikeda, H. J. Org. Chem. 1999, 64, 387.

[8] Breslow, R.; Zhang, X.; Xu, R.; Maletic, M.; Merger, R. J. Am. Chem. Soc. 1996, 118, 11678.

[9] Fujita, K.; Ishizu, T.; Minamiura, N.; Yamamoto, T. Chem. Lett. 1991, 1889.

[10] Khan, A. R.; Forgo, P.; Stine, K. J.; D'Souza, V. T. Chem. Rev. 1998, 98, 1977.

[11] Zhang, H.-C.; Liu, Z.-N.; An, W.; Hao, A.-Y.; Sun, L.-Z. Chin. J. Org. Chem. 2010, 30, 1279 (in Chinese).

(张华承, 刘召娜, 安伟, 郝爱友, 孙立臻, 有机化学, 2010, 30, 1279.)

[12] Zhang, Y.; Li, L.-S.; Cheng, B.-P. Chin. J. Anal. Chem. 2014, 42, 375 (in Chinese). (张杨, 李来生, 程彪平, 分析化学, 2014, 42, 375.)

[13] Ren, X. J.; Xue, Y.; Liu, J. Q.; Zhang, D.; Zheng, J.; Luo, G. M.; Guo, C. H.; Mu, Y.; Shen, J. C. ChemBioChem 2002, 3, 356.

[14] Martinez, A.; Mellet, C. O.; Fernandez, J. M. G. Chem. Soc. Rev. 2013, 42, 4746.

[15] Zhou, R.-D.; Li, L.-S.; Cheng, B.-P.; Nie, G.-Z.; Zhang, H.-F. Acta Chim. Sinica 2014, 72, 720 (in Chinese).

(周仁丹, 李来生, 程彪平, 聂桂珍, 张宏福, 化学学报, 2014, 72, 720.)

[16] Liu, Y.; You, C.-C.; Zhang, H.-Y. Supramolecular Chemistry-Molecular Recognition and Assembly of Synthetic Receptors, Nankai University Press, Tianjin, 2001, pp. 241 242 (in Chinese). (刘育, 尤长城, 张衡益, 超分子化学——合成受体的分子识别 与组装, 天津, 南开大学出版社, 2001, pp. 241 242.)

[17] Benesi, H. A.; Hidebrand, J. H. J. Am. Chem. Soc. 1949, 71, 2703.

[18] Liu, Y.; You, C.-C.; Chen, Y.; Wada, T.; Inoue, Y. J. Org. Chem. 1999, 64, 7781 7787 .

[19] Liu, Y.; Chen, Y.; Li, B.; Wada, T.; Inoue, Y. Chem. Eur. J. 2001, 7, 2528.

[20] Li, L; Li, X.-Y.; Liu, Y. Chin. Sci. Bull. 2003, 48, 2442 (in Chinese). (李莉, 李晓云, 刘育, 科学通报, 2003, 48, 2442.)

[21] Zhao, Y; Yang, Z.-M.; Zhu, H.-Y. Acta Phys.-Chim. Sin. 2007, 23, 394 (in Chinese). (赵炎, 杨自明, 朱洪友等, 物理化学学报, 2007, 23, 394.)

[22] Cabrer, P. R.; Alvarez-Parrilla, E.; Meijida, F.; Seijas, J. A.; Ntifiez, E. R.; Zato, J. V. Langmuir 1999, 15, 5489. 